\title{
A DENSITY THEOREM FOR LACUNARY FOURIER SERIES
}

\author{
BY J. W. MOELLER AND P. O. FREDERICKSON
}

Communicated by A. Zygmund, June 28, 1965

I. Introduction. Let $\Lambda$ be a nonempty subset of the integers, and let $L^{2}(\Lambda)$ denote the closed subspace of $L^{2}(0,2 \pi)$ spanned by the exponentials $\left\{e^{i \lambda x} \mid \lambda \in \Lambda\right\}$. Suppose we are given the values of an arbitrary function $f$ in $L^{2}(\Lambda)$ on a fixed interval of positive length $\delta$. When can we determine the values of $f$ outside that interval? A precise answer to this question will be announced below, after some essential terminology has been introduced to help us handle the problem.

Accordingly, let $\chi_{\delta}$ denote the indicator function for the interval $(0, \delta)$, and let $A_{\delta}(f)=\chi_{\delta} f$; in words, $A_{\delta}(f)$ is simply the function which coincides with $f$ on the interval $(0, \delta)$ but vanishes elsewhere. We say that a set of integers $\Lambda$ is an extrapolation set of length $\rho$ if the mapping $A_{\delta}: L^{2}(\Lambda) \rightarrow \chi_{\delta} L^{2}(\Lambda)$ has a bounded inverse for $\delta>\rho$ but fails to have a bounded inverse whenever $\delta<\rho$. It is easy to see that every set of integers has a unique extrapolation length $\rho$, and $A_{\delta}^{-1}$ will extrapolate functions in $L^{2}(\Lambda)$ from $(0, \delta)$ onto $(0,2 \pi)$ as long as $\delta>\rho$. Of course, since $L^{2}(\Lambda)$ is translation invariant, there is nothing sacred about our choice of the interval $(0, \delta)$; any other interval of length $\delta$ would serve the same purpose.

It turns out that the extrapolation length of a prescribed set can be explicitly computed if we know how sparsely the points in this set are distributed. The appropriate concept to use in this connection is the notion of uniform outer density. Following Kahane [2], we define the uniform outer density of a set $\Lambda$ to be

$$
\lim _{\alpha \rightarrow \infty} \frac{1}{\alpha}\left\{\sup _{-\infty<\sigma<\infty} N(\sigma, \alpha)\right\},
$$

where $N(\sigma, \alpha)$ represents the number of points of $\Lambda$ contained in the interval $[\sigma, \sigma+\alpha)$. Our main result expresses the exact relationship between outer density and extrapolation length.

THEOREM. Let $\Lambda$ be a set of integers whose uniform outer density is $D(\Lambda)$. Then $\Lambda$ is an extrapolation set of length $\rho$ if and only if $\rho=2 \pi D(\Lambda)$.

A detailed proof of this Theorem, further generalized to include $L^{2}$ spaces of exponential functions with gaps in their spectra, will be published elsewhere [7]. In what follows we briefly outline our plan of attack to expose the main ideas. 
II. Exponential subspaces. Fix an arbitrary set of integers $\Lambda$, choose a number $\alpha>0$, and let $E_{\alpha}$ denote the closed subspace of $L^{2}(0, \infty)$ spanned by the exponentials $\left\{e^{-(\alpha+i \lambda) x} \mid \lambda \in \Lambda\right\}$. For any $g \in L^{2}(0, \infty)$ and any $h>0$, let $\left(T^{h} g\right)(x)$ denote the restriction of $g(x+h)$ to the non-negative reals, and let $T_{\alpha}^{h}$ denote the restriction of $T^{h}$ to the subspace $E_{\alpha}$. Obviously $T^{h}\left(E_{\alpha}\right) \subset E_{\alpha}$ for all $h \geqq 0$ because $E_{\alpha}$ is generated by exponentials. Using $L^{2}$ norms, we now define

$$
\left\|T_{\alpha}^{h}\right\|=\sup _{o \in E_{\alpha}} \frac{\left\|T^{h} g\right\|}{\|g\|} \quad(g \neq 0) .
$$

As the following lemmas suggest, this quantity will play an important role in our subsequent work.

Lemma 1. If $\left\|T_{\beta}^{h}\right\|=1$ for some $\beta>0$, then $\left\|T_{\alpha}^{h}\right\|=1$ for all $\alpha>0$.

Lemma 2. A necessary and sufficient condition for $\Lambda$ to be an extrapolation set of length $\rho$ is that

$$
\sup \left\{h \mid\left\|T_{\alpha}^{h}\right\|=1\right\}=\rho .
$$

LEMMA 3. If $\Lambda$ is an extrapolation set of length $\rho$, then $\lim _{\alpha \rightarrow \infty}\left\|T_{\alpha}^{h}\right\|=0$ whenever $h>\rho$.

Each of these results may be proved by arguments which are quite elementary [6]; altogether, they reduce the problem of computing extrapolation length to one of estimating an operator norm. Such an estimate has already been obtained by Lax [4], who derived the fundamental inequality

$$
\left\|T_{\alpha}^{h}\right\|^{2} \leqq\left(1-\theta_{\alpha}(\tau)\right)\left(1-e^{-2 h r}\right)^{-1},
$$

where

$$
\theta_{\alpha}(\tau)=\inf _{-\infty<\sigma<\infty}\left|B_{\alpha}(\sigma+i \tau)\right|
$$

and

$$
B_{\alpha}(s)=\prod_{\lambda \in \Lambda} \frac{s+\lambda-i \alpha}{s+\lambda+i \alpha} \cdot \frac{\lambda+i \alpha}{\lambda-i \alpha} \quad(s=\sigma+i \tau) .
$$

It is possible to secure asymptotically sharp upper and lower bounds for $\theta_{\alpha}(\tau)$ with the help of a useful lemma due to Koosis. The proof of a similar result may be found in [3].

Lemma 4. Let $\sigma$ and $\tau$ be real numbers, $\tau>0$, and let w be any complex number with Im $w>0$. Then, for any $\epsilon>0$, there is $a \delta<0$ such that 


$$
\frac{\tau(2-\epsilon)}{|w-\sigma|^{2}} \operatorname{Im} w \leqq \log \frac{|\sigma+i \tau-\tilde{w}|}{|\sigma+i \tau-w|} \leqq \frac{\tau(2+\epsilon)}{|w-\sigma|^{2}} \operatorname{Im} w
$$

whenever $|\tau /(w-\sigma)|<\delta$.

Corollary 1. If the condition

$$
\limsup _{\alpha \rightarrow \infty}\left\{\sup _{\sigma} \sum_{\lambda \in \Lambda} \frac{2 \alpha}{\alpha^{2}+(\lambda+\sigma)^{2}}\right\} \leqq \rho
$$

is satisfied, it follows that $\left\|T_{\alpha}^{h}\right\|<1$ for all $h>\rho$.

Lemma 5. Let $w$ be any complex number such that Im $w>0$, and let $d_{\alpha}(w)$ denote the distance from the space $E_{\alpha}$ to the normalized exponential function $(2 \operatorname{Im~} w)^{1 / 2} e^{i w x}$. Then $d_{\alpha}(w)=\left|B_{\alpha}\left(w^{*}\right)\right|$, where the asterisk denotes negative complex conjugation.

The distance formula cited above [5] actually implies the converse of Corollary 1. To see this, fix a positive value of $\tau$ and decompose the normalized exponential functions $(2 \tau)^{1 / 2} e^{-(\tau+i \sigma) x}$ by orthogonal projection into $E_{\alpha}$ so that

$$
(2 \tau)^{1 / 2} e^{-(r+i \sigma) x}=k_{\alpha}(\sigma ; x)+f_{\alpha}(\sigma ; x),
$$

where $f_{\alpha} \in E_{\alpha}$ and $k_{\alpha}$ is orthogonal to $E_{\alpha}$. Now apply $T^{h}$ to both sides of this equation and take norms to obtain the inequality

$$
e^{-\tau h} \leqq\left\|T^{h} f_{\alpha}\right\|+\left\|T^{h} k_{\alpha}\right\| \text {. }
$$

Since $\left\|T^{h}\right\| \leqq 1$, it follows from Lemma 5 that

$$
e^{-\tau h} \leqq\left\|T^{h} f_{\alpha}\right\|+\left|B_{\alpha}(\sigma+i \tau)\right| .
$$

Consequently, if $\left\|T_{\alpha}^{n}\right\|<1$, an application of Lemma 3 at once yields

$$
\limsup _{\alpha \rightarrow \infty}\left\{\sup _{\sigma}\left|B_{\alpha}^{-1}(\sigma+i \tau)\right|\right\} \leqq e^{\tau h} .
$$

Corollary 2. If $\left\|T_{\alpha}^{h}\right\|<1$ for all $h>\rho$, then

$$
\limsup _{\alpha \rightarrow \infty}\left\{\sup _{\sigma} \sum_{\lambda \in \Lambda} \frac{2 \alpha}{\alpha^{2}+(\lambda+\sigma)^{2}}\right\} \leqq \rho .
$$

III. A density relation. The summation appearing in Corollary 1 and Corollary 2 has a number theoretic interpretation which was first conjectured by Professor J.-P. Kahane for the case $\rho=0$. Our proof that Kahane's conjecture is true in general rests on the next lemma. 
LEMma 6. Let $\Lambda$ be an arbitrary set of integers having outer uniform density equal to $D(\Lambda)$. Then, for any integer $k>0$ and any positive $\epsilon$, there exists a number $l \geqq 1$ having the property that there are $k$ consecutive intervals of length $l$, each of which contains at least $(D(\Lambda)-\epsilon) l$ points of $\Lambda$.

If we define $\Omega_{j}(k)$ to be the interval $[j \alpha / k,(j+1) \alpha / k)$ and take

$$
M(\alpha)=\sup _{\sigma} N(\sigma, \alpha) \text {, }
$$

it follows that

$$
\sup _{\sigma} \sum_{\lambda \in \Lambda} \frac{\alpha}{\alpha^{2}+(\lambda+\sigma)^{2}} \leqq M(\alpha / k) \sum_{j} \sup _{\lambda \in \Omega_{j}(k)}\left(\frac{\alpha}{\alpha^{2}+\lambda^{2}}\right) .
$$

Using the well-known formula [1]

$$
\frac{\pi \sinh 2 \pi k}{\cosh 2 \pi k-1}=\frac{1}{k}+2 \sum_{j=1}^{\infty} \frac{k}{j^{2}+k^{2}},
$$

we may pass to the limit as $\alpha \rightarrow \infty$ and then as $k \rightarrow \infty$ to conclude that

$$
\limsup _{\alpha \rightarrow \infty}\left\{\sup _{\sigma} \sum \frac{\alpha}{\alpha^{2}+(\lambda+\sigma)^{2}}\right\} \leqq \pi D(\Lambda) \text {. }
$$

An application of Lemma 6 and a similar argument now suffice to establish the reverse inequality

$$
\limsup _{\alpha \rightarrow \infty}\left\{\sup _{\sigma} \sum \frac{\alpha}{\alpha^{2}+(\lambda+\sigma)^{2}}\right\} \geqq \pi(D(\Lambda)-\epsilon) .
$$

This completes the proof of our main theorem.

Acknowledgments. We wish to express our thanks to Professors J.-P. Kahane, P. Koosis, and P. D. Lax for their valuable suggestions.

Added in Proof. We recently learned that Professor Kahane has obtained the principal theorem of this note in a more general setting with different methods. However, our techniques can be extended without difficulty and may be of some interest in themselves, since we rely mostly upon simple properties of the shift operator to secure our results.

\section{BIBLIOGRAPHY}

1. H. Cartan, Elementary theory of analytic functions of one or several complex variables, Addison-Wesley, Reading, Mass., 1963.

2. J.-P. Kahane, Lacunary Taylor and Fourier series, Bull. Amer. Math. Soc. 70 (1964), 199-213. 
3. P. Koosis, Interior compact spaces of functions on a half-line, Comm. Pure Appl. Math. 10 (1957), 583-615.

4. P. D. Lax, Remarks on the preceding paper of Koosis, Comm. Pure Appl. Math. 10 (1957), 617-622.

5. - Translation invariant spaces, Acta Math. 101 (1959), 163-178.

6. J. W. Moeller, On the extrapolation of Lacunary Fourier series, J. Reine Angew. Math. (to appear).

7. - Sums of exponential functions determined by their values on an interval, J. Math. Anal. Appl. (to appear).

Case Institute of Technology

\section{ON THE EQUATION $f^{n}+g^{n}=1$}

BY FRED GROSS

Communicated by Walter Rudin, July 23, 1965

There is a close relationship between Fermat's last theorem and the family of solutions $f$ and $g$ of the functional equation

$$
x^{n}+y^{n}=1 \text {. }
$$

If, for example, $S_{D}$ denotes the class of all pairs $(f, g)$ of single valued functions $f$ and $g$ meromorphic in a domain $D$ and having the additional property that, for some $z_{0}$ in $D, f\left(z_{0}\right)$ and $g\left(z_{0}\right)$ are both positive rationals, then either, for $n>2$, (1) has no solutions in $S_{D}$ or Fermat's last theorem is not true.

In this note we discuss the solutions of (1) meromorphic in the complex plane. We shall call such solutions $M_{c}$ solutions.

Theorem 1. For $n=2$, all $M_{c}$ solutions are of the form

$$
f=\frac{2 \beta(z)}{1+\beta(z)^{2}} \quad \text { and } \quad g(z)=\frac{1-\beta(z)^{2}}{1+\beta(z)^{2}} .
$$

Proof. This follows directly from a theorem on uniformization [1]. We need only note that for $n=2,(1)$ is of genus zero and that the rational solution (2), with $\beta(z)=z$, maps the whole $z$-plane in a 1-1 manner on the Riemann surface of (1).

THEOREM 2. For $n=3, M_{c}$ solutions exist. One such solution is given by:

$$
\begin{aligned}
& f=4^{-1 / 6}\left(\wp^{\prime}\right)^{-1}\left(1+3^{-1 / 2} \cdot 4^{1 / 8} \wp\right), \\
& g=4^{-1 / 6}(\wp)^{-1}\left(1-3^{-1 / 2} \cdot 4^{1 / 8} \wp\right),
\end{aligned}
$$

where is a Weierstrass $\$$-function. 